\title{
Bivalent mTOR inhibitors - the next generation
}

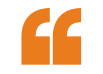

xenografts

that were

resistant

to either

rapamycin

or to TORKi

retained full

sensitivity to

RapaLink-1

The phosphoinositide 3-kinaseAKT-mechanistic target of rapamycin (PI3K-AKT-mTOR) pathway is one of the most commonly activated pathways in human cancer, and a number of agents have been developed against various nodes of the pathway. For mTOR, these comprise the natural product rapamycin and its analogues (rapalogs), as well as mTOR kinase inhibitors (TORKi), which are currently in clinical trials. Reporting in Nature, the groups of Shokat and Rosen have now examined the resistance mechanisms to first- and second-generation mTOR inhibitors, and they have

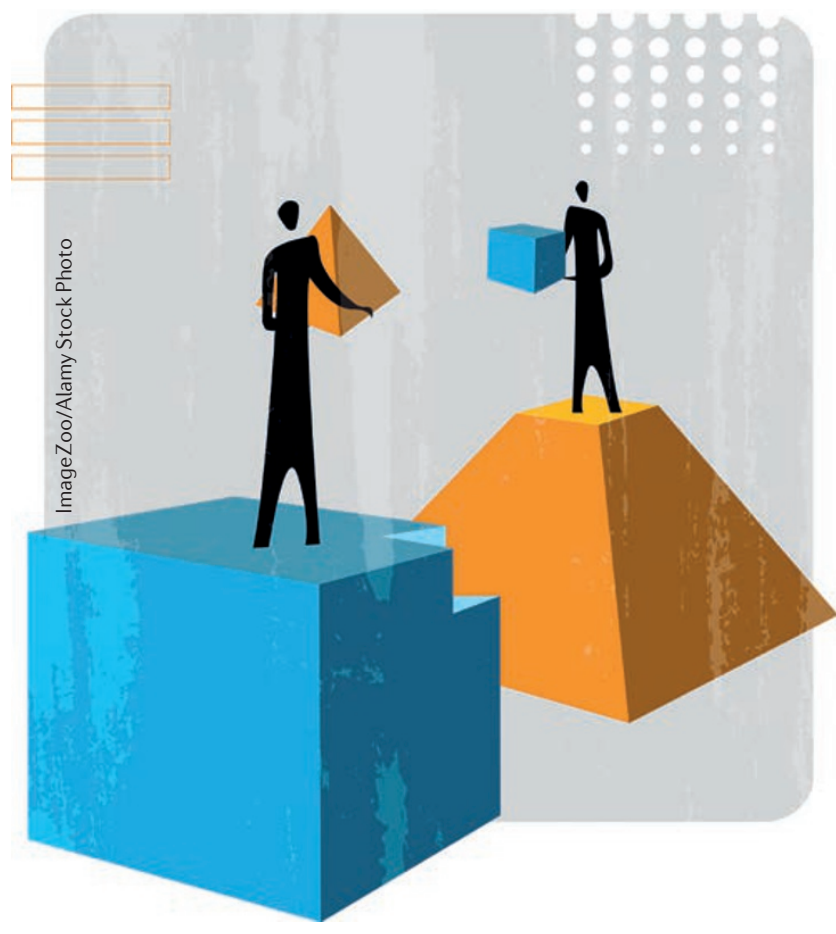

used this information to inform the development of third-generation mTOR inhibitors.

In order to map mTOR resistance mutations, the authors exposed the MCF-7 breast cancer cell line to either rapamycin or the TORKi AZD8055 for 3 months, until resistant colonies emerged. Deep sequencing of two clones of rapamycin-resistant cells revealed mutations in the FKBP12rapamycin binding (FRB) domain, whereas the clone of AZD8055resistant cells had a mutation in the kinase domain of mTOR.

The causative nature of these mutations for resistance was confirmed in biochemical experiments.

Screens in yeast had previously shown that that single amino acid changes in the FRB domain can confer rapamycin resistance through steric hindrance. By contrast, the mutation that caused resistance to AZD8055 was found to map to a residue more than $15 \AA$ away from the TORKi binding site. This mutation was found to hyperactivate the kinase and thereby confer a growth advantage to the cells. This mutation, as well other mutations that map to the mTOR kinase domain, had previously been identified in untreated patients with cancer - highlighting the need for a new class of inhibitors capable of targeting both pre-existing mutations as well as emerging ones.

Using molecular modelling, the authors found that the rapamycinbinding and TORKi-binding sites are juxtaposed. This inspired the idea of an avidity-based approach to overcome drug resistance to both first- and second-generation mTOR inhibitors. They selected the highly specific TORKi MLN0128, which is currently in clinical trials, and connected it to rapamycin with numerous different linkers. One of the resulting bivalent molecules, RapaLink-1, was found to potently inhibit the growth of MCF-7 cells at levels comparable to rapamycin alone and to a combination of rapamycin with MLN1028.

In in vivo experiments, RapaLink-1 (administered to mice by intraperitoneal injection) showed similar potency to current clinical mTOR inhibitors, and xenografts that were resistant to either rapamycin or to TORKi retained full sensitivity to RapaLink-1. The authors also constructed cells with doublemutant mTOR that were resistant to rapamycin, MLN0128 and to a combination of the two. However, these cells remained as sensitive to RapaLink-1 treatment as cells with wild-type mTOR — indicating that RapaLink-1 represents a new class of mTOR inhibitors that can overcome resistance to the first two generations of mTOR inhibitors.

\section{Alexandra Flemming}

ORIGINAL ARTICLE Rodrik-Outmezguine, V. S. et al. Overcoming mTOR resistance mutations with a new-generation mTOR inhibitor. Nature 534, 272-276 (2016) 